\title{
Implementing New Approaches to Tuberculosis Control
}

\author{
Kristine M. Guinn'; Eric J. Rubin ${ }^{1, *}$
}

As someone who has studied tuberculosis (TB) for many years, it is difficult for me to admit, but tuberculosis has not been the most exciting field in medicine for a long time. The vaccine that is used in much of the world was developed in the 1930s. The foundational clinical research that defines how we treat TB was performed in India in the 1960s. And the last new drug to enter the $\mathrm{TB}$ armamentarium was introduced in many countries almost 30 years ago.

This slow pace has had an important consequence. Over the last few decades the emphasis in public health has moved from new therapies to doing a better job of implementing what we already have. This means that basic public health measures, including large-scale diagnostics, contact tracing, drug supply logistics and ensuring that patients are able to complete long treatment courses, have become the leading edge in efforts to control TB.

But changes are on the horizon, with both challenges and opportunities emerging in $\mathrm{TB}$ intervention. New diagnostics, new treatments and, potentially, new ways to prevent TB have the potential of dramatically changing how we approach disease, both at the level of the individual patient and also for the uninfected public. But employing these new tools is going to require reinvention at the level of implementation. And they must be applied in a landscape of significant and increasing drug resistant disease. Thus, the challenge of the next several years is not only going to be in developing tools but also determining how we're going to use them to produce real change.

\section{THE PROBLEM OF DRUG RESISTANCE}

Antibiotic resistance in TB is hardly a new problem. Very early after the introduction of streptomycin patients were seen to fail monotherapy. And, since the disease is transmissible, resistance could easily spread in the population. That problem could be mitigated, to some extent, using combination therapy. In theory, this could limit the evolution of new drug resistance as combinatorial selection makes escape mutations much less likely.

However, practice has not kept up with theory. As each new antibiotic was developed, it was introduced into populations that had already serially acquired individual resistance mutations to the existing drugs. This was exacerbated by inappropriate prescribing, inconsistent drug supply and imperfect patient compliance all leading to increased development and subsequent transmission of drug resistant TB. To make matters worse, many countries and localities have little ability to detect drug resistance. In the most recent World Health Organization (WHO) report (1), of 10 million worldwide incident $\mathrm{TB}$ cases, 465,000 are thought to be multidrug resistant. This is a frightening number but is also undoubtedly an underestimate. Because drug resistance is either detected late or not at all, many patients are being treated inadequately, resulting in greater morbidity and mortality and unchecked transmission of resistant strains.

\section{BETTER DIAGNOSTICS}

For close to a century we've relied on two approaches to definitively diagnose TB. Sputum smears are fast but relatively insensitive while culture is highly sensitive but extremely slow. Fortunately, this has changed dramatically in recent years. The most dramatic results have come from culture-independent methodologies that result from the combination of engineering, genetics, and biochemistry such as the GeneXpert MTB (2) and TrueNat MTB (3) devices. These have two substantial advantages. They are very rapid and can provide answers directly from sputum. And these tests can not only diagnose disease but can simultaneously predict drug resistance. These tests come on the heels of other technical improvements, including the growing use of broth culture methods which can also accelerate growth.

Of course, these new technologies are only useful if they are actually used. New devices and the supplies they require are prohibitively expensive for most endemic parts of the world even after international subsidies. Reference labs that perform reliable culture- 
based testing, which is essential for quality control in TB control programs, are not widely available. As multidrug- and extensively drug-resistant TB become more widespread, traditional drug susceptibility testing for second- and third-line drugs becomes even more important. Yet labs capable of reliably testing for these antibiotic susceptibilities, like the excellent lab at the Chinese CDC, are unavailable in most of the world. And, finally, how the information generated in the lab gets translated into practice remains problematic.

\section{MORE EFFECTIVE THERAPIES AND TREATMENT STRATEGIES}

The current therapeutic strategy for TB has served us fairly well for a long time. In controlled circumstances, cure rates are very high, 90\%, at least for drug-susceptible disease. Unfortunately, real-world results still translate to an estimated $>1.2$ million annual deaths (1). There are many reasons for this. Patients are lost all along the "TB care cascade," with many never diagnosed, others never getting connected to care following their diagnosis, others not having access to drugs, and still others starting but not completing therapy (4). There is no question that the cumbersome and extended multidrug course of therapy is a contributor to our frustratingly high failure rates. In addition, rising drug resistance rates mean that many patients receiving standard therapy are being inadequately treated with very high failure rates.

The problem with current therapy is two-fold. The length of therapy means that a substantial infrastructure needs to be built to ensure compliance - though infrastructure is expensive and, as shown by failure rates, doesn't always work. And our treatments for drug resistant disease are typically even longer, with higher failure rates.

Over the past few years we have seen real changes, however. Several new antibiotics have entered the TB armamentarium. For most of these, there is no real pre-existing resistance, so they are active against circulating drug resistant Mycobacterium tuberculosis (Mtb) strains. Three novel classes of antituberculous drugs are now available and more are on the horizon. Bedaquiline (5), an inhibitor of the mycobacterial ATP synthase, is a very potent drug. Relative resistance is rare, though there is cross-resistance with the third-line agent clofazimine so that there is some pre-existing resistance ( $(6)$. Two nitroimidazoles, delamanid ( 7 ) and pretomanid (8), have nearly identical mechanisms but pre-existing resistance is rare if it exists at all. And linezolid, an oxazolidinone drug, is not new but has been shown to be useful for drug-resistant disease (9). Because it is not widely used, pre-existing linezolid resistance is uncommon. Using these drugs in combination with existing second- and third-line agents has resulted in much higher cure rates for drugresistant TB. And, most intriguingly, an open label clinical trial showed that using only three oral drugs, bedaquiline, pretomanid and linezolid, resulted in high cure rates within six months in patients with either extensively- (XDR) or multidrug-resistant (MDR) TB who had failed or could not tolerate other regimens (10). These regimens hold great promise for many patients, many of whom were previously untreatable.

For several years, preclinical animal studies have suggested that there might be treatments that could result in a shorter course of treatment for drugsusceptible disease. Until this year, however, clinical trials have been disappointing. But a recently announced trial (unpublished as of the date of writing) has shown that a standard regimen that includes rifapentine instead of the rifampin together with added moxifloxacin for four months with directly observed therapy is non-inferior to the standard six month regimen. And animal experiments that test combinations including new drugs could make that even shorter (11). Given the potency of the recently released agents, it might even be possible to arrive at a "universal regimen" that could simultaneously be shorter and be used to treat all patients regardless of drug resistance. Given the toxicity of some of the newer drugs, particularly linezolid, more work will have to be done before we get there, but it remains an aspirational goal.

\section{THE POSSIBILITY OF PREVENTION}

Most of the world, including China, administers the Mycobacterium bovis BCG (BCG) vaccine. This agent, developed in the 1930s, helps to prevent the devastating consequences of childhood $\mathrm{TB}$ but has little efficacy in controlling TB in adults. There has been a long search for a better vaccine that could offer protection to adults but, until recently, trials have been disappointing. However, over the past two years, two studies have shown at least some promise. One suggested that revaccination of adolescents with BCG might protect against persistent $\mathrm{Mtb}$ infection (12). Another used a new adjuvant together with a fusion of two Mtb protein antigens, had efficacy of $50 \%$ in preventing progression of infection to disease over the 
course of three years (13).

These new strategies are still far from clinical application. But they do suggest that it is possible to achieve better protection in adults than is afforded by BCG. Having even a partially-effective vaccine could change the TB control landscape, though how much of a change it would bring about would entirely depend on its performance characteristics.

\section{THE CHALLENGE OF IMPLEMENTATION}

The early experience with rolling out vaccination against COVID-19 has brought one aspect of disease control into sharp relief - the vaccine is only as good as the implementation strategy that utilizes it. This lesson has long been clear in TB control. Right now, we have good diagnostics and effective and inexpensive drugs, even if they can be improved. But they are being implemented inconsistently around the world, resulting in a huge number of preventable deaths. Having a strong TB control program is the best weapon we have both to ensure proper treatment of individuals and also, because treatment is our best form of prevention, protecting public health.

But rapid change is a challenge to even the best TB control programs. After all, one of the reasons that that these programs can be successful is that they are standardized, ensuring the same care to all patients. However, some of the new technologies require much more individualization, particularly when it comes to drug-resistant disease. And changing even standardized approaches can be very difficult in large and bureaucratic organizations. In fact, many of us have heard from programs that would prefer to stick with older paradigms even when new interventions are demonstrably superior.

Because there are so many new aspects of diagnosis and treatment, it is now time to embrace change and make it an integral part of TB control. Measures that might help include:

- Algorithms that account for the varying speed and sensitivity of newer diagnostics. It would be simpler if any given country adopted a new diagnostic modality at all nationwide sites simultaneously. This will not happen. Instead, TB controllers are going to be faced with different types of information on each patient at each site. They will need help in understanding how to interpret these tests and what each finding should trigger for treatment and contact tracing.

- Rapid recognition of drug resistance. Earlier appropriate treatment is associated with decreased transmission but, in many programs, resistance isn't recognized until clinical failure occurs. Finding patients with drug resistance not only benefits them but has important consequences for public health.

- An ability to rapidly incorporate advances in therapeutics. WHO guidelines are changing rapidly and the pace of change is likely to accelerate. Not all drugs are equally accessible in all parts of the world. But some of these hold the promise of having a significant impact on individuals and populations. TB control programs should be planning for how they will change rather than simply responding to new guidelines when they arise.

- Creating structures for individualized therapy. Identifying and optimally treating drug resistant TB requires a separate pathway within control programs. The best programs not only diagnose drug resistance early but also create individualized treatment regimens based on the specific resistance profiles of isolates. This can be accomplished either through expert guidance for each individual or, perhaps more practically, through predefined algorithms that change as new information becomes available. In fact, most clinical trials have been performed with patients with varying degrees of drug resistance, enabling the creation of appropriate algorithms.

- Integration or, at least, coordination with HIV treatment programs. Patients, particularly those with low CD4 counts, benefit from starting HIV treatment early when TB is diagnosed. And a single program is best suited to monitor drugdrug interactions between antiretrovirals and $\mathrm{TB}$ antibiotics.

We live in an exciting time for TB control. After decades of incremental changes we are on the edge of substantive advancements in how we approach the identification, treatment and prevention of TB. But they will only make a difference if we can apply them across the spectrum of translation, all the way from the lab to the public health program.

doi: $10.46234 / \mathrm{ccdcw} 2021.053$

\# Corresponding author: Eric J. Rubin, erubin@hsph.harvard.edu.

\footnotetext{
${ }^{1}$ Department of Immunology and Infectious Diseases, Harvard T. H. Chan School of Public Health, Boston, Massachusetts, USA.
} 
Submitted: February 07, 2021; Accepted: February 19, 2021

\section{REFERENCES}

1. World Health Organization. Global tuberculosis report. 2020. https://apps.who.int/iris/bitstream/handle/10665/336069/9789240013 131-eng.pdf. [2021-2-7].

2. Boehme CC, Nabeta P, Hillemann D, Nicol MP, Shenai S, Krapp F, et al. Rapid molecular detection of tuberculosis and rifampin resistance. $\mathrm{N}$ Engl J Med 2010;363(11):1005 - 15. http://dx.doi.org/10.1056/ NEJMoa0907847.

3. Nikam C, Jagannath M, Narayanan MM, Ramanabhiraman V, Kazi M, Shetty A, et al. Rapid diagnosis of Mycobacterium tuberculosis with truenat MTB: a near-care approach. PLoS One 2013;8(1):e51121. http://dx.doi.org/10.1371/journal.pone.0051121.

4. Naidoo P, Theron G, Rangaka MX, Chihota VN, Vaughan L, Brey $\mathrm{ZO}$, et al. The South African tuberculosis care cascade: estimated losses and methodological challenges. J Infect Dis 2017;216(S7):S702 - 13. http://dx.doi.org/10.1093/infdis/jix335.

5. Diacon AH, Pym A, Grobusch MP, de los Rios JM, Gotuzzo E, Vasilyeva I, et al. Multidrug-resistant tuberculosis and culture conversion with bedaquiline. N Engl J Med 2014;371(8):723 - 32. http://dx.doi.org/10.1056/NEJMoa1313865.

6. Nimmo C, Millard J, van Dorp L, Brien K, Moodley S, Wolf A, et al. Population-level emergence of bedaquiline and clofazimine resistanceassociated variants among patients with drug-resistant tuberculosis in southern Africa: a phenotypic and phylogenetic analysis. Lancet Microbe 2020;1(4):e165 - 74. http://dx.doi.org/10.1016/S2666-5247 (20)30031-8.
7. Gupta R, Gao MQ, Cirule A, Xiao HP, Geiter LJ, Wells CD. Delamanid for extensively drug-resistant tuberculosis. N Engl J Med 2015;373(3):291 - 2. http://dx.doi.org/10.1056/NEJMc1415332.

8. Dawson R, Diacon AH, Everitt D, van Niekerk C, Donald PR, Burger DA, et al. Efficiency and safety of the combination of moxifloxacin, pretomanid (PA-824), and pyrazinamide during the first 8 weeks of antituberculosis treatment: a phase $2 \mathrm{~b}$, open-label, partly randomised trial in patients with drug-susceptible or drug-resistant pulmonary tuberculosis. Lancet 2015;385(9979):1738 - 47. http://dx.doi.org/10. 1016/S0140-6736(14)62002-X.

9. Lee M, Lee J, Carroll MW, Choi H, Min S, Song T, et al. Linezolid for treatment of chronic extensively drug-resistant tuberculosis. N Engl J Med 2012;367(16):1508 - 18. http://dx.doi.org/10.1056/NEJMoa1 201964.

10. Conradie F, Diacon AH, Ngubane N, Howell P, Everitt D, Crook AM, et al. Treatment of highly drug-resistant pulmonary tuberculosis. N Engl J Med 2020;382(10):893 - 902. http://dx.doi.org/10.1056/ NEJMoa1901814.

11. Xu J, Li SY, Almeida DV, Tasneen R, Barnes-Boyle K, Converse PJ, et al. Contribution of pretomanid to novel regimens containing bedaquiline with either linezolid or moxifloxacin and pyrazinamide in murine models of tuberculosis. Antimicrob Agents Chemother 2019;63(5):e00021 - 19. http://dx.doi.org/10.1128/AAC.00021-19.

12. Nemes E, Geldenhuys H, Rozot V, Rutkowski KT, Ratangee F, Bilek $\mathrm{N}$, et al. Prevention of $M$. tuberculosis infection with H4:IC31 vaccine or BCG revaccination. N Engl J Med 2018;379(2):138 - 49. http://dx.doi.org/10.1056/NEJMoa1714021.

13. Tait DR, Hatherill M, van der Meeren O, Ginsberg AM, van Brakel E, Salaun B, et al. Final analysis of a trial of $\mathrm{M} 72 / \mathrm{AS} 01_{\mathrm{E}}$ vaccine to prevent tuberculosis. N Engl J Med 2019;381(25):2429 - 39. http://dx.doi.org/ 10.1056/NEJMoa1909953.
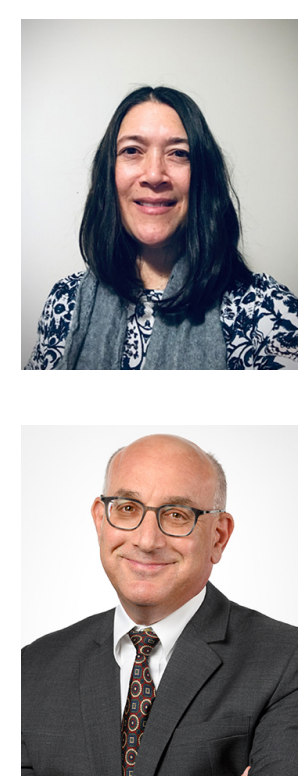

Kristine M. Guinn

Project Manager, Harvard T.H. Chan School of Public Health

Eric J. Rubin

Professor, Harvard T.H. Chan School of Public Health Editor-in-Chief, New England Journal of Medicine 